\title{
Determinación de la Actividad Antioxidante, contenido de Fenoles Totales, Taninos Totales y Flavonoides Totales del Hidromiel de Sauco (sambucus peruviana) de Cuatro Empresas del Distrito de Talavera en el año 2019
}

Determinants of the Antioxidant activity, content of Total Phenols, Total Tannins and Total Flavonoids of elder mead (sambucus peruviana) from four Companies in the Talavera District in the year 2019

\author{
Dr. Jorge Wilmer Elías Silupu a; jelias s@hotmail.es \\ Universidad Nacional Ciro Alegría \\ Mg. Ronald Pérez Salcedo ${ }^{\boldsymbol{b}}$ ronaldps 13@gmail.com \\ Universidad Nacional José María Arguedas \\ Mg. Celia Rocio Yauris Silvera ${ }^{c}$; celyauris13@gmail.com \\ Universidad Nacional José María Arguedas \\ Mg. Javier Alejandro Manrique Catalán ${ }^{c}$; manrique c@hotmail.com \\ Universidad Nacional Ciro Alegría
}

Recibido: Enero / 08 /2021-Revisado: Febrero / 10 /2021-Publicado: Marzo /31 / 2021

\section{RESUMEN}

En esta investigación el objetivo principal fue determinar la actividad antioxidante, contenido de fenoles totales, taninos totales y flavonoides totales del hidromiel de saúco (Sambucus peruviana) de cuatro empresas del distrito de Talavera en el año 2019. En consecuencia, se utilizó el método espectrofotométrico con el reactivo DPPH para determinar la actividad antioxidante; con respecto al contenido de fenoles totales con Folin-Ciocalteau; para el contenido de taninos totales se utilizó ácido clorhídrico y para el contenido de flavonoides totales se analizó con el reactivo de tricloruro de aluminio. Por otro lado, los resultados se obtuvieron haciendo un análisis de datos y comparación mediante el DCA que fueron tabulados y evaluados a través del análisis de varianza (ANOVA), mediante la comparación prueba de rango múltiple se determinó la significancia de las medias con un nivel de confianza del $95 \%$, empleando diferencias mínimas significativas (LSD), donde mostraron el (valor- $P<5 \%$ ) esto explica que se rechazó la hipótesis nula, donde se demostraron que existen diferencias estadísticamente significativas de la actividad antioxidante, el contenido de fenoles totales, taninos totales y flavonoides totales del hidromiel de sauco de cuatro empresas del distrito de Talavera. Donde la empresa Santa Isabel presenta el mayor nivel de actividad antioxidante del hidromiel de sauco (2.830 $\pm 0.003 \mathrm{mM}$ Eq. trolox/100 $\mathrm{mL}$ de hidromiel de sauco), la empresa El Dorado tiene mayor contenido de fenoles totales (3783.38 $\pm 2.33 \mathrm{mg}$ equivalente de ácido gálico (AGE)/ $100 \mathrm{~mL}$ de hidromiel de sauco), la empresa Santa Isabel obtuvo el mayor contenido de taninos totales (12.254 \pm $0.022 \mathrm{mg} / \mathrm{L}$ de hidromiel de sauco) y la empresa Marqués de Aranjuez muestra mayor contenido de flavonoides totales (335.46 $\pm 0.00 \mathrm{mg}$ equivalente de quercetina/ $\mathrm{mL}$ de hidromiel de sauco). Finalmente, se concluye que la empresa Santa Isabel presenta el mayor nivel de actividad antioxidante del hidromiel de sauco, la empresa El Dorado tiene mayor contenido de fenoles totales, la empresa Santa Isabel obtuvo el mayor contenido de taninos totales y la empresa Marqués de Aranjuez muestra mayor contenido de flavonoides del hidromiel de sauco.

Palabras claves: Actividad antioxidante, fenoles, taninos, flavonoides, hidromiel y sauco. 


\begin{abstract}
In this research work the main objective was to determine the antioxidant activity, phenol content Total, total tannins and total flavonoids of elder mead (Sambucus peruviana) from four companies in the Talavera district in 2019. Consequently, the spectrophotometric method with the DPPH reagent was used to determine the antioxidant activity; regarding the content of total phenols with Folin-Ciocalteau; for the total tannin content, hydrochloric acid was used and for the total flavonoid content it was analyzed with the aluminum trichloride reagent. On the other hand, the results were obtained by doing a data analysis and comparison by means of the DCA that were tabulated and evaluated through the analysis of variance (ANOVA), using the multiple range test comparison the significance of the means with a level was determined. of $95 \%$ confidence, using significant minimum differences ( $L S D)$, where they showed the (value- $P<\alpha$ ) this explains that the null hypothesis was rejected, where it was shown that there are statistically significant differences in antioxidant activity, the content of phenols Total, total tannins and total flavonoids of elder mead from four companies in the Talavera district. When that the company Santa Isabel has the highest level of antioxidant activity of elder mead (2.830 $\pm 0.003 \mathrm{mM}$ Eq. Trolox $/ 100 \mathrm{~mL}$ of mead), El Dorado has a higher total phenolic content (3783.38 $\pm 2.33 \mathrm{mg}$ equivalent of gallic acid (AGE) / $100 \mathrm{~mL}$ of mead, the Santa Isabel company obtained the highest total tannin content $(12,254 \pm 0.022 \mathrm{mg} / \mathrm{L}$ of elder mead) and the company Marqués de Aranjuez shows higher total flavonoid content (335,46 $\pm 0.00 \mathrm{mg}$ equivalent of quercetin / $\mathrm{mL}$ of elder mead). Finally, it is concluded that the company Santa Isabel has the highest level of antioxidant activity of elder mead, El Dorado has a higher total phenolic content, the Santa Isabel company obtained the highest total tannin content and the company Marqués de Aranjuez shows higher total flavonoid content of elder mead.
\end{abstract}

Keywords: Antioxidant activity, phenols, tannins, flavonoids, mead and elder

\title{
Introducción
}

El hidromiel de sauco es una bebida alcohólica obtenida mediante fermentación por levaduras, agua y jugo de sauco, ya que este producto es elaborado por las empresas de Marqués de Aranjuez, Santa Isabel, Granja Santa Rosa y El Dorado. Las propiedades beneficiosas del hidromiel de sauco aparentemente se deben a su actividad antioxidante y por su contenido de fenoles totales, taninos y flavonoides totales. Ya que siempre en un producto busca la calidad de hidromiel y está relacionada con la presencia de los taninos. Los taninos del hidromiel proceden principalmente de las semillas y los hollejos de sauco transferidos al hidromiel durante su elaboración. Por ello, estas bebidas alcohólicas 
cuyo mosto están en contacto con dichas partes de sauco, por lo cual su consumo puede prevenir las enfermedades crónicas asociadas al estrés oxidativo como diabetes, enfermedades cardiovasculares, cáncer, entre otros. (Aguilar, 2015)

Los fenoles totales son de gran importancia en la enología debido al papel que juegan directa o indirectamente sobre las características del hidromiel, en gran parte a la concentración y composición de los fenoles. Estos compuestos son los responsables del color, astringencia, amargor y aroma de los hidromieles. (Flanzy, 2003, p. 783)

Los antioxidantes son compuestos fenólicos que inhiben o retrasan el proceso oxidativo de otras moléculas y la mayoría de antioxidantes naturales se obtienen a partir de plantas y son mayoritariamente compuestos fenólicos 0 polifenólicos. Llobera (2004). Los componentes fenólicos juegan un rol importante en el color y sabor de los alimentos y bebidas; su consumo ha sido asociado con efectos benéficos para la salud humana. Jáuregui et al. (2007) por otro lado, los flavonoides tienen importantes propiedades antioxidantes, ya que minimizan la peroxidación lipídica y efecto de radicales libres, contribuyendo esta manera a reducir el riesgo de enfermedades cardiovasculares. Soto (2007).

Por ello, en la presente investigación se determinó la actividad antioxidante, contenido de fenoles totales, taninos totales y flavonoides totales del hidromiel de saúco (Sambucus peruviana) de cuatro empresas del distrito de Talavera en el año 2019.Apurímac.

\section{Materiales y métodos}

\section{Equipos}

- Espectrofotómetro UV-Visible.

- Refrigeradora.

- Agitador magnético digital.

- Termómetro digital desde $-50^{\circ} \mathrm{C}$ hasta $+200^{\circ} \mathrm{C}$.

- Estufa. 
-Balanza electrónica digital.

Reactivos e insumos

- Reactivo de Folin-Ciocalteu Sigma 2N

- Ácido clorhídrico $37.0 \%$ de pureza

- Metanol químicamente puro al $80 \%$

- Agua destilada aproximadamente $\mathrm{pH}: 7$

- Etanol 95 GL; alcohol etílico

-DPPH (2, 2-difenil-1-picrilhidrazil), al $90 \%$

- Carbonato de Sodio 99,9 \% de pureza

- Ácido gálico $99 \%$ de pureza

- Quercetina $95 \%$ de pureza

- Tricloruro de aluminio $98 \%$ de pureza.

\section{Métodos.}

a. Método de análisis desarrollado por. Brand-Williams et al. (1995) para la determinación de la actividad antioxidante con la siguiente ecuación.

Se restaron las absorbancias del blanco y de la muestra, y se calculó mM equivalentes Trolox/mL como se muestra en la Ec. (01).

mMEq. $\frac{\text { Trolox }}{\mathrm{mL}}$ muestra $=\frac{\mathrm{Abs}_{\text {blanco }}-\mathrm{Abs}_{\text {muestra }}}{\mathrm{m} *(\mathrm{~mL} \text { de muestra }) * 1000} \ldots$

Dónde: $\mathrm{m}=$ pendiente de la curva calibrada,

$$
\begin{aligned}
& \mathrm{mM}=\text { Milimolar } \\
& \mathrm{Eq}=\text { Equivalente }
\end{aligned}
$$

b. Método de análisis desarrollado por. Singleton et al. (1999), para la determinación del contenido de fenoles totales: Índice de Folin Ciocalteu con la siguiente ecuación. 
El contenido de fenoles totales se estimó a partir de la curva estándar con una solución acuosa de ácido gálico como patrón.

La curva estándar de calibración para la determinación de fenoles totales se muestra en la siguiente Ec. (02).

$\mathrm{Y}=\mathrm{a} A B S+b$

Fenoles totales $=\frac{\mathrm{Y} \times \mathrm{D} \times \mathrm{V} \times 100}{\text { Peso }}$

Dónde, fenoles totales $=$ Miligramos $(\mathrm{mg})$ equivalente de ácido gálico (GAE) /100 $\mathrm{mL}$ muestra,

$$
\mathrm{Y}=\text { Miligramos (mg) equivalente de ácido gálico (GAE)/ por }
$$

$\mathrm{mL}$,

$$
\begin{aligned}
& \mathrm{D}=\text { Dilución, } \mathrm{V}=\text { Volumen de muestra en } \mathrm{mL} \text {, } \\
& \text { Peso = Peso de la muestra en } \mathrm{mL} \text {. }
\end{aligned}
$$

\section{c. Método de análisis desarrollado por (Bate, 1981) para la determinación del contenido de taninos totales con la siguiente ecuación.}

Para la determinación del contenido de taninos totales se trabajó utilizando la siguiente Ec. (04).

C. T. T. $(\mathrm{mg} / \mathrm{L}$ de muestra $)=19.33 \times\left(A_{A}-A_{B}\right)$. . Ec. $(04)$

- Dónde, $\mathbf{A}_{\mathbf{A}}=$ Absorbancia en tubo "A", $\mathbf{A}_{\mathbf{B}}=$ Absorbancia en tubo " $\mathrm{B}$ "

- $\mathrm{mg}=$ Miligramos, $\mathrm{L}=\mathrm{Litro}, \mathrm{La}$ constante 19.33 corresponde al coeficiente de extinción molar de la cianidina obtenida por la hidrólisis ácida de una disolución de procianidinas oligómeras de referencia (Ribéreau-Gayon, et al.,1998). 
d. Método de análisis desarrollado por. Hamasaka et al. (2004) para la determinación del contenido de flavonoides totales con la siguiente ecuación.

Para la determinación del contenido de flavonoides totales se trabajó utilizando la siguiente Ec. (05).

$\mathrm{FI} .=(\operatorname{Am} \times \operatorname{Pr} \times 5) / \operatorname{Ar} \times 100$

Donde, $\mathrm{FI}=$ Contenido de flavonoides totales expresados como $\mathrm{mg}$ equivalente de quercetina $(E Q) / m L$,

Am = Absorbancia de la solución muestra $(\mathrm{nm})$,

$\operatorname{Pr}=$ Peso de la sustancia de referencia $(\mathrm{g})$ y

$\mathrm{Ar}=$ Absorbancia de la solución de referencia $(\mathrm{nm})$.

\section{Resultado y discusión}

Resultados y discusiones de la actividad antioxidante del hidromiel de sauco

Resultados de la actividad antioxidante

La actividad antioxidante se calculó usando una curva estándar de calibración de Trolox, y los resultados se expresan como mM equivalente de Trolox $/ 100 \mathrm{~mL}$ de hidromiel. Se determinó del promedio de tres repeticiones, considerando la absorbancia a $515 \mathrm{~nm}$.

Tabla 1. Resultados de la actividad antioxidante del hidromiel de sauco

\begin{tabular}{ll}
\hline $\begin{array}{l}\text { Hidromiel de sauco de las empresas } \\
\text { del distrito de Talavera }\end{array}$ & $\begin{array}{l}\text { Actividad antioxidante (mM } \\
\text { Eq.trolox } / \mathbf{1 0 0} \mathbf{~} \mathbf{L} \text { de hidromiel de } \\
\text { sauco) } \\
(\overline{\mathbf{x}} \pm \mathbf{S})\end{array}$ \\
\hline Marqués de Aranjuez & $2.718(\mathrm{a}) \pm 0.004$ \\
Santa Isabel & $2.830(\mathrm{~b}) \pm 0.003$ \\
Granja Santa Rosa & $2.683(\mathrm{c}) \pm 0.005$
\end{tabular}




\section{El Dorado}

$$
2.259(d) \pm 0.005
$$

\section{$\overline{\mathrm{x}}$ : Promedio de 3 repeticiones S: Desviación estándar}

Así mismo se observa los resultados de la actividad antioxidante de cuatro empresas del distrito de Talavera en la tabla 1. Donde la empresa Santa Isabel presenta mayor nivel de la actividad antioxidante con $(2.830$ (b) $\pm 0.003 \mathrm{mM}$ Eq. trolox / $100 \mathrm{~mL}$ de hidromiel de sauco) que Marqués de Aranjuez (2.718 (a) \pm 0.004 mM Eq. trolox / $100 \mathrm{~mL}$ de hidromiel de sauco), seguido por Granja Santa Rosa (2.683 (c) $\pm 0.005 \mathrm{mM}$ Eq. trolox / $100 \mathrm{~mL}$ de hidromiel de sauco y El Dorado (2.259 (d) $\pm 0.005 \mathrm{mM}$ Eq. trolox / $100 \mathrm{~mL}$ de hidromiel de sauco).

Los resultados obtenidos por triplicado, fueron tabulados y evaluados a través del análisis de varianza (ANOVA), donde el ( $p$ - value $<5 \%$ ) de nivel de significancia entonces se rechaza la hipótesis nula por lo tanto se acepta la hipótesis alterna, que la actividad antioxidante del hidromiel de sauco presentaron una diferencia significativa con un nivel de confianza al $95 \%$ y mediante la comparación de medias de la actividad antioxidante del hidromiel de sauco de cuatro empresas del distrito de talavera como se observan en la figura 1.

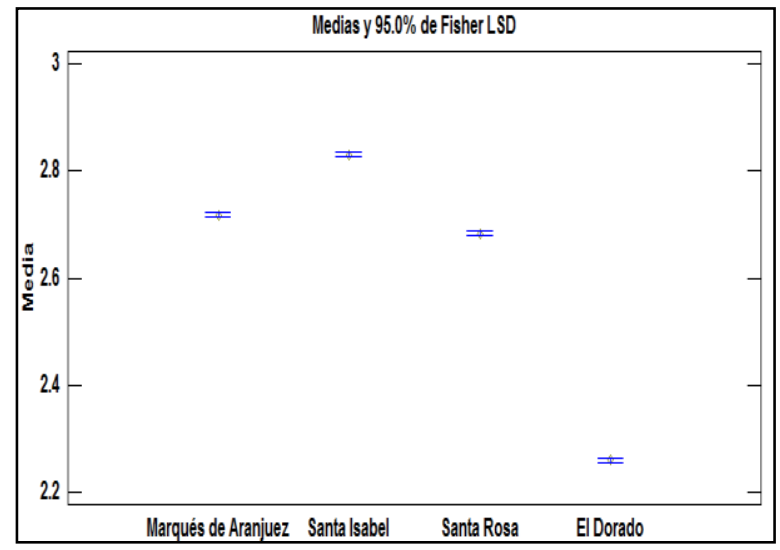

Figura 1. Comparación de medias para actividad antioxidante con LSD Fisher

En la figura 1 se observa la comparación de medias de la actividad antioxidante con LSD de las empresas de Marqués de Aranjuez, Santa Isabel, Granja Santa Rosa y empresa El Dorado donde muestran diferencias estadísticamente 
significativas entre sí, con un nivel del $95.0 \%$ de confianza, siendo la empresa Santa Isabel presenta mayor nivel de actividad antioxidante del hidromiel de sauco.

\section{Discusión de resultado de la actividad antioxidante}

La empresa Santa Isabel presenta mayor nivel de actividad antioxidante con (2.830 (b) $\pm 0.003 \mathrm{mM} \mathrm{Eq.} \mathrm{trolox} \mathrm{/} 100 \mathrm{~mL}$ de hidromiel de sauco) que Marqués de Aranjuez (2.718 (a) $\pm 0.004 \mathrm{mM}$ Eq. trolox / $100 \mathrm{~mL}$ de hidromiel de sauco), seguido por Granja Santa Rosa (2.683 (c) $\pm 0.005 \mathrm{mM}$ Eq. trolox $/ 100 \mathrm{~mL}$ de hidromiel de sauco y El Dorado (2.259 (d) $\pm 0.005 \mathrm{mM} \mathrm{Eq}$. Trolox $/ 100 \mathrm{~mL}$ de hidromiel de sauco). Los resultados se compararon al reporte de (Leyva, 2009) la actividad antioxidante de licor de mora presenta los valores de $110.51 \pm 0.18$ y $304.47 \pm 2.65 \mathrm{mM}$ equivalentes de trolox/100 mL de licor, es fue mayor actividad antioxidante que hidromiel de sauco de cuatro empresas, según los reportes de (Alén et al., 2009) los valores de actividad antioxidante determinados por el método DPPH en los vino tinto Sabater con $4.22 \pm 0.65 \mathrm{mM}$ Eq.trolox $/ 100 \mathrm{~mL}$, vino tinto Escursac con $3.27 \pm$ 0.29, mM Eq.trolox /100 mL, vino tinto Gorgollassa con $3.42 \pm 0.51 \mathrm{mM}$ Eq.trolox $/ 100 \mathrm{~mL}$, vino blanco Giró Ros con $0.33 \pm 0.06 \mathrm{mM}$ Eq.trolox /100 mL y vino blanco Quigat con $0.31 \pm 0.07 \mathrm{mM}$ Eq.trolox $/ 100 \mathrm{~mL}$, por otro lado la capacidad antioxidante de los vinos analizados en este estudio se encuentran en los rangos reportados por (Fernández et al., 2004) (Tintos: 2.33 a 7.85 mM Eq.trolox /100 mL, Blancos: 0.08 a 1. $45 \mathrm{mM}$ Eq.trolox /100 mL, (Landrault et al., 2001) en un trabajo realizado para evaluar la actividad antioxidante de vinos franceses reportan valores promedio para vinos tintos $18.29 \mathrm{mM}$ Eq.trolox $/ 100 \mathrm{~mL}$, vinos blancos $3.36 \mathrm{mM}$ Eq.trolox /100 mL, vinos rosados $3.2 \mathrm{mM}$ Eq.trolox $/ 100 \mathrm{~mL}$ y vino tinto Tempranillo $15.4 \mathrm{mM}$ Eq.trolox /100 mL. Los resultados obtenidos de la capacidad antioxidante en diferentes tipos de vinos realizado por (Simonetti et al.,1997) señala valores promedio de $12.3 \mathrm{mM}$ Eq.trolox / $100 \mathrm{~mL}$ y $14.916 \mathrm{mM}$ Eq.trolox / $100 \mathrm{~mL}$ en vinos tintos Italianos y Sur Africanos.

\section{Resultados del contenido de fenoles totales del hidromiel de sauco}




\section{Resultados del contenido de fenoles totales}

El contenido de fenoles totales se estimó a partir de una curva estándar de calibración de ácido gálico como patrón. Los resultados se expresaron como mg equivalente de ácido gálico (AGE)/ $100 \mathrm{~mL}$ de hidromiel, se determinó por triplicado, considerando la absorbancia a $765 \mathrm{~nm}$.

Tabla 2. Resultados del contenido de fenoles totales del hidromiel

de sauco

\begin{tabular}{ll}
\hline $\begin{array}{l}\text { Hidromiel de sauco de las } \\
\text { empresas del distrito de }\end{array}$ & $\begin{array}{l}\text { Contenido de fenoles totales (mg } \\
\text { equivalente de ácido gálico (AGE)/ } 100 \\
\text { Talavera }\end{array}$ \\
$\begin{array}{ll}\mathbf{m L} \text { de hidromiel de sauco) }( \\
\mathbf{x} \pm \mathbf{S})\end{array}$ \\
\hline Marqués de Aranjuez & $3635.94(\mathrm{a}) \pm 2.27$ \\
Santa Isabel & 3759.35 (b) \pm 0.86 \\
Granja Santa Rosa & $3714.62(\mathrm{c}) \pm 8.92$ \\
El Dorado & 3783.38 (d) \pm 2.33 \\
\hline
\end{tabular}

$\overline{\mathrm{x}}$ : Promedio de 3 repeticiones S: Desviación estándar

En la tabla 2 se muestran resultados del contenido de fenoles totales del hidromiel de sauco de cuatro empresas del distrito de Talavera, donde la empresa El Dorado tiene mayor contenido de fenoles totales con (3783.38 (d) $\pm 2.33 \mathrm{mg}$ equivalente de ácido gálico (AGE)/ $100 \mathrm{~mL}$ de hidromiel de sauco) que Santa Isabel (3759.35 (b) $\pm 0.86 \mathrm{mg}$ equivalente de ácido gálico (AGE)/ $100 \mathrm{~mL}$ de hidromiel de sauco), seguido por Granja Santa Rosa (3714.62 (c) $\pm 8.92 \mathrm{mg}$ equivalente de ácido gálico (AGE)/ $100 \mathrm{~mL}$ de hidromiel de sauco) y Marqués de Aranjuez (3635.94 (a) \pm $2.27 \mathrm{mg}$ equivalente de ácido gálico (AGE)/ $100 \mathrm{~mL}$ de hidromiel de sauco).

Los resultados obtenidos por triplicado, fueron tabulados y evaluados a través del análisis de varianza (ANOVA), Donde el valor-P es menor que $5 \%$ de nivel de significancia entonces se rechaza la hipótesis nula por lo tanto se acepta la hipótesis alterna, el contenido de fenoles totales del hidromiel de sauco presentaron 
una diferencia significativa con un nivel de confianza al $95 \%$ y mediante la comparación de medias del contenido de fenoles totales del hidromiel de sauco de cuatro empresas del distrito de talavera como se observa en la figura 2.

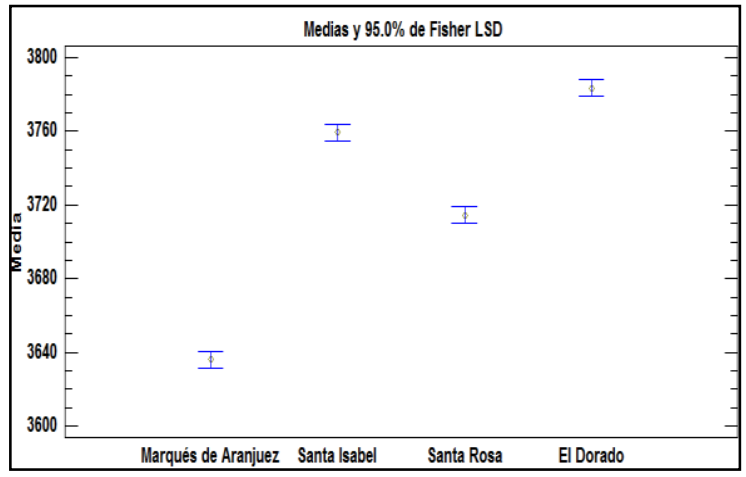

Figura 2. Comparación de medias para el contenido de fenoles totales con LSD Fisher.

En la figura 2 se observa la comparación de medias del contenido de fenoles totales con LSD de las empresas de Marqués de Aranjuez, Santa Isabel, Granja Santa Rosa y El Dorado donde muestran diferencias estadísticamente significativas entre sí, con un nivel del 95.0 \% de confianza, siendo la empresa El Dorado tiene mayor contenido de fenoles totales del hidromiel de sauco.

\section{Discusión de resultado del contenido de fenoles totales}

En la tabla 2 se muestran los resultados del contenido de fenoles totales en función de la cantidad equivalente de ácido gálico, los resultados muestran que la empresa que tiene mayor contenido de fenoles totales fue El Dorado con (3783.38 $\pm 2.33 \mathrm{mg}$ equivalente de ácido gálico (AGE)/ $100 \mathrm{~mL}$ de hidromiel de sauco) y la empresa Santa Isabel tiene (3759.35 \pm 0.86 mg equivalente de ácido gálico (AGE)/ $100 \mathrm{~mL}$ de hidromiel de sauco), seguido por la Granja Santa Rosa (3714.62 \pm 8.92 mg equivalente de ácido gálico (AGE)/ $100 \mathrm{~mL}$ de hidromiel de sauco) y el de menor contenido de fenoles totales es de la empresa Marqués de Aranjuez (3635.94 \pm 2.27 mg equivalente de ácido gálico (AGE)/ $100 \mathrm{~mL}$ de hidromiel de sauco). A comparación de los resultados según al reporte del (Leyva, 2009) el contenido de 
fenoles totales de licor de mora se encuentra en un intervalo de $477.12 \pm 0.04 \mathrm{y}$ $884.98 \pm 0.03 \mathrm{mg} \mathrm{EAG} / 100 \mathrm{~mL}$ de licor. Los resultados obtenidos de (Alén et al., 2009), de polifenoles totales vino tinto Sabater con $1169140 \pm 2.06 \mathrm{mg}$ ácido gálico/100 mL, vino tinto Escursac con $971400 \pm 2.81 \mathrm{mg}$ ácido gálico/ $100 \mathrm{~mL}$, vino tinto Gorgollassa con 869590 × 3.58 mg ácido gálico/ 100 mL, vino blanco Giró Ros con $214860 \pm 0.94 \mathrm{mg}$ ácido gálico/ $100 \mathrm{~mL}$ y vino blanco Quigat con $128020 \pm 0.31$ $\mathrm{mg}$ ácido gálico/ $100 \mathrm{~mL}$, Los resultados obtenidos según (Jáuregui et al., 2007), contenido de compuestos fenólicos en vinos producidos en Perú se estimó usando el método Folin-Ciocalteau, obteniéndose valores de 627000 a 3321000 mg GAE/100 mL. Por su parte (Avalos et al., 2003), evaluó el contenido fenoles totales expresados en $\mathrm{mg}$ equivalente de ácido gálico (AGE)/100 mL, que estuvo comprendido en el rango de 85.33 a 180.33 para vinos tintos. Por otro lado, un estudio realizado por (Muñoz et al., 2007), en el cual se analizó el contenido fenoles totales de 13 tipos de vinos peruanos elaborados en los departamentos de Ica y lima, obtuvo concentraciones entre 8627000 a $3321000 \mathrm{mg} / 100 \mathrm{~mL}$ de fenólicas totales, mientras que (Salazar et al., 2011), observación en otros vinos peruanos entre 2370000 a $3610000 \mathrm{mg} / 100 \mathrm{~mL}$, siendo los vinos la Casona del Valle (Borgoña) y Santiago Queirolo (Tannat).

\section{Resultados del contenido de taninos totales del hidromiel de sauco}

\section{Resultados del contenido de taninos totales}

Los resultados se expresaron como $\mathrm{mg} / \mathrm{L}$ de hidromiel de sauco se determinó por triplicado, considerando la absorbancia a $555 \mathrm{~nm}$.

Tabla 3. Resultados del contenido de taninos totales del hidromiel de sauco

\begin{tabular}{ll}
\hline $\begin{array}{l}\text { Hidromiel de sauco de las empresas } \\
\text { del distrito de Talavera }\end{array}$ & $\begin{array}{l}\text { Contenido de taninos totales }(\mathbf{m g} / \mathbf{L} \\
\text { de hidromiel de sauco) } \\
(\overline{\mathbf{x}} \pm \mathbf{S})\end{array}$ \\
\hline Marqués de Aranjuez & $7.679(\mathrm{a}) \pm 0.178$ \\
Santa Isabel & $12.254(\mathrm{~b}) \pm 0.022$
\end{tabular}




\section{Granja Santa Rosa}

El Dorado
11.836 (c) \pm 0.233

$10.576(d) \pm 0.024$

$\overline{\mathrm{x}}$ : Promedio de 3 repeticiones S: Desviación estándar

Los resultados del contenido de taninos totales del hidromiel de sauco de cuatro empresas del distrito de Talavera muestran en la tabla 3. Donde la empresa Santa Isabel tiene mayor contenido de taninos totales con $(12.254$ (b) $\pm 0.022 \mathrm{mg} / \mathrm{L}$ de hidromiel de sauco) que Granja Santa Rosa (11.836 (c) $\pm 0.233 \mathrm{mg} / \mathrm{L}$ de hidromiel de sauco), seguido por El Dorado (10.576 (d) $\pm 0.024 \mathrm{mg} / \mathrm{L}$ de hidromiel de sauco) y Marqués de Aranjuez (7.679 (a) $\pm 0.178 \mathrm{mg} / \mathrm{L}$ de hidromiel de sauco).

Los resultados obtenidos por triplicado, fueron tabulados y evaluados a través del análisis de varianza (ANOVA), Donde el valor-P es menor que 0.05 de nivel de significancia entonces se rechaza la hipótesis nula por lo tanto se acepta la hipótesis alterna, entonces el contenido de taninos totales del hidromiel de sauco presentaron una diferencia significativa con un nivel de confianza al $95 \%$ y mediante la comparación de medias del contenido de taninos totales del hidromiel de sauco de cuatro empresas del distrito de talavera como se observa en la figura 3.

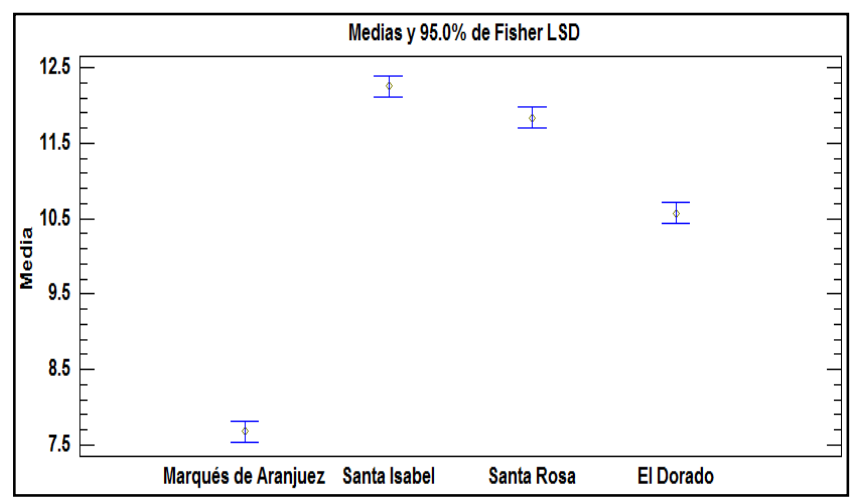

Figura 3. Comparación de medias para el contenido de taninos totales con LSD Fisher.

En la figura 3 se observa la comparación de medias del contenido de taninos totales con LSD de las empresas de Marqués de Aranjuez, Santa Isabel, Granja Santa Rosa y El Dorado donde muestran diferencias estadísticamente significativas 
entre sí, con un nivel del $95.0 \%$ de confianza, siendo la empresa Santa Isabel tiene mayor contenido de taninos del hidromiel de sauco.

\section{Discusión de resultado del contenido de taninos totales}

En la tabla 3 se muestra el contenido de taninos totales, los resultados muestran que la empresa Santa Isabel tiene mayor contenido de taninos totales con (12.254 $\pm 0.022 \mathrm{mg} / \mathrm{L}$ de hidromiel de sauco) mientras la empresa Granja Santa Rosa (11.836 $\pm 0.233 \mathrm{mg} / \mathrm{L}$ de hidromiel de sauco), y la empresa El Dorado (10.576 $\pm 0.024 \mathrm{mg} / \mathrm{L}$ de hidromiel de sauco) siendo el Marqués de Aranjuez con el menor contenido de taninos totales $(7.679 \pm 0.178 \mathrm{mg} / \mathrm{L}$ de hidromiel de sauco). En comparación a los reportes de (Frankel et al., 1995) la presencia de taninos totales en el vino tinto seco de 0,93 ( $\mathrm{mg} / \mathrm{L}$ de muestra), en vino tinto semi seco de 0,85 ( $\mathrm{mg} / \mathrm{L}$ de muestra) y en vino rosé semi seco de 0,62 ( $\mathrm{mg} / \mathrm{L}$ de muestra), los resultados expuestos por (Narváez, 2010), quien comparó tres de taninos totales en 20 vinos tintos chilenos concentraciones que variaron entre 0.4 a $1.7 \mathrm{mg} / \mathrm{L}$. El contenido de taninos totales en esta investigación fue de vinos blancos con maceración ( 0.37 a $0.08 \mathrm{mg} / \mathrm{L}$ ); para vinos blancos con 0.61 a $0,16 \mathrm{mg} / \mathrm{L}$; vinos tintos con 2.29 a $0.96 \mathrm{mg} / \mathrm{L}$ y vinos rosados con 0.28 a $0.68 \mathrm{mg} / \mathrm{L}$, (Rodríguez y García, 2005) al igual que en este trabajo los autores reportan el contenido de taninos totales en los vinos tintos $(3.36 \mathrm{mg} / \mathrm{L})$ comparados con rosados $(0.37 \mathrm{mg} / \mathrm{L})$ y vinos blancos $(0,19 \mathrm{mg} / \mathrm{L})$, señalado por (Durán y Trujillo, 2008) el contenido de taninos $t$ evaluado en este estudio se encuentra en el rango 0 - 70 a $3 \mathrm{~g} \mathrm{mg} / \mathrm{L}$ en una muestra de vinos tintos, Los resultados según (Arteaga et al., 2016) indican el contenido de taninos aportados al vino por la uva que oscilan entre 4,968 como valor máximo y $0,213 \mathrm{mg} / \mathrm{L}$ como valor mínimo.

Resultados del contenido de flavonoides totales del hidromiel de sauco Resultados del contenido de flavonoides totales 
El contenido de flavonoides totales se calculó usando una curva estándar de calibración de quercetina y los resultados se expresaron como mg equivalente de quercetina $/ \mathrm{mL}$ de hidromiel de sauco (Anexo7). Se determinó por triplicado, considerando la absorbancia a $420 \mathrm{~nm}$ (Anexo 4).

Tabla 4. Resultados del contenido de flavonoides totales del hidromiel de sauco

\section{Hidromiel de sauco de las empresas del distrito de}

Talavera
Contenido de flavonoides totales ( $\mathrm{mg}$ equivalente de quercetina $/ \mathrm{mL}$ de hidromiel de sauco)

$(\overline{\mathrm{x}} \pm \mathrm{S})$
Marqués de Aranjuez

Santa Isabel

Granja Santa Rosa

El Dorado
335.46 (a) \pm 0.00

335.35 (b) \pm 0.00

335.31 (c) \pm 0.00

335.39 (d) \pm 0.00

$\overline{\mathrm{x}}$ : Promedio de 3 repeticiones S: Desviación estándar

Los resultados del contenido de flavonoides totales de las cuatro empresas del distrito de Talavera se observan en la siguiente tabla 4. Donde la empresa Marqués de Aranjuez tiene mayor contenido de flavonoides totales con (335.46 (a) $\pm 0.00 \mathrm{mg}$ equivalente de quercetina $/ \mathrm{mL}$ de hidromiel de sauco) que El Dorado (335.39 (d) $\pm 0.00 \mathrm{mg}$ equivalente de quercetina $/ \mathrm{mL}$ de hidromiel de sauco), seguido por Santa Isabel (335.35 (b) $\pm 0.00 \mathrm{mg}$ equivalente de quercetina $/ \mathrm{mL}$ de hidromiel de sauco) y Granja Santa Rosa (335.31 (c) $\pm 0.00 \mathrm{mg}$ equivalente de quercetina/mL de hidromiel de sauco).

Los resultados obtenidos por triplicado, fueron tabulados y evaluados a través del análisis de varianza (ANOVA), Donde el valor-P es menor que 0.05 de nivel de significancia entonces se rechaza la hipótesis nula por lo tanto se acepta la hipótesis alterna, donde el contenido de flavonoides totales del hidromiel de sauco presentaron una diferencia significativa con un nivel de confianza al $95 \%$ y mediante 
la comparación de medias del contenido de flavonoides totales del hidromiel de sauco de cuatro empresas del distrito de talavera como se observa en la figura 4.

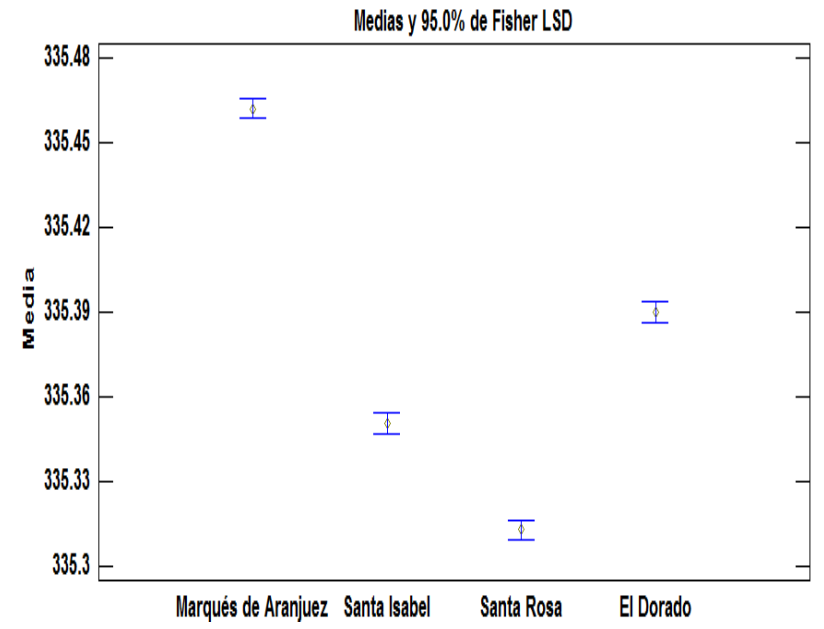

Figura 4. Comparación de medias para el contenido de flavonoides totales con LSD Fisher.

En la figura 4 se observa la comparación de medias del contenido de flavonoides totales con LSD de las empresas de Marqués de Aranjuez, Santa Isabel, Granja Santa Rosa y El Dorado donde muestran diferencias estadísticamente significativas entre sí, con un nivel del 95.0 \% de confianza, siendo la empresa la empresa El Dorado tiene mayor contenido de flavonoides totales del hidromiel de sauco.

\section{Discusión de resultado del contenido de flavonoides totales}

En cuanto al resultados del contenido de flavonoides totales de la empresa que tiene mayor contenido fue El Marqués de Aranjuez que presenta un (335.46 \pm $0.00 \mathrm{mg}$ equivalente de quercetina/ $\mathrm{mL}$ de hidromiel de sauco) respecto a la empresa El Dorado tiene $(335.39 \pm 0.00 \mathrm{mg}$ equivalente de quercetina $/ \mathrm{mL}$ de hidromiel de sauco), con una diferencia mínima le sigue la empresa Santa Isabel con un (335.35 $\pm 0.00 \mathrm{mg}$ equivalente de quercetina $/ \mathrm{mL}$ de hidromiel de sauco) y por último la Granja Santa Rosa (335.31 $\pm 0.00 \mathrm{mg}$ equivalente de quercetina/mL de hidromiel de sauco). Estos resultados obtenidos en comparación a otros 
reportes fueron así como reporta según (lanzy, 2003) los vinos tintos poseen una concentración de flavonoides promedio de $17500 \mathrm{mg} / \mathrm{mL}$, por su parte (Muñoz, 2006) en su estudio en vinos cv. Carménère, la concentración promedio de flavonoides fue de $26900 \mathrm{mg} / \mathrm{mL}$ en el Valle del Maule, En un estudio realizado por (Ketter, 2008) en vinos comerciales cv Cabernet sauvignon de las vendimias 2002 y 2003 en el Valle del Maipo, la concentración promedio de flavonoides fue de $21800 \mathrm{mg} / \mathrm{mL}$, mientras que (Radovanovie, 2010) la concentración de flavonoides totales estuvo en el rango de 1870000 a 3139000 mg/mL, con el vino Tacama presentando el mayor contenido de dichos compuestos, por (Rodrigo y Candy, 2011) mientras que por espectrofotometría se determinó la cantidad flavonoides totales (1869190 a $3138850 \mathrm{mg} / \mathrm{mL})$ de 8 vinos peruanos contenidos aromáticos.

\section{Conclusiones}

1. Se determinó que la empresa Santa Isabel presenta el mayor nivel de actividad antioxidante del hidromiel de saúco (Sambucus peruviana), a diferencia del Marqués de Aranjuez, seguido por la Granja Santa Rosa y el que tiene menor actividad antioxidante es la empresa El Dorado.

2. El contenido de fenoles totales se determinó mediante espectrofotometría llegando a la conclusión que la empresa que tiene mayor contenido de fenoles totales del hidromiel de saúco (Sambucus peruviana) fue El Dorado y la empresa Santa Isabel, seguido por la Granja Santa Rosa y contenido de fenoles totales de la empresa Marqués de Aranjuez fueron menores.

3. Con respecto al contenido de taninos totales de acuerdo a la investigación realizada se obtuvo los siguientes resultados, la empresa Santa Isabel tiene mayor contenido de taninos totales del hidromiel de saúco (Sambucus peruviana) mientras la empresa Granja Santa Rosa, y la empresa EI Dorado siendo el Marqués de Aranjuez con el menor contenido de taninos totales.

4. Se determinó el contenido de flavonoides totales, llegando a la conclusión que la empresa Marqués de Aranjuez destacó por su alto contenido de flavonoides totales del hidromiel de saúco (Sambucus peruviana), respecto 
a la empresa El Dorado, con una diferencia mínima le sigue la empresa Santa Isabel y por último la Granja Santa Rosa.

\section{Referencias}

Aguilar, T. (2015). Comparación de la capacidad antioxidante de mostos y vinos tintos del valle del Itata, Chile. Departamento de Agroindustrias, facultada de ingeniería Agricula, universidadde concepción, chillan, chile.

Alén-Ruiz, F. G.-F.-L.-C.-G. (2009). "influence of major polyphenols on antioxidant activity in mencía and brancellao red wines". food chemistry, vol. 113, pgs. 53 $-60$.

Arteaga S., C. J. (2016). Análisis comparativo del contenido de taninos en vinos comerciales de Tarija, Bolivia. Ciencia Sur Vol. 2. № 2. ISSN 2518 - 4792, Pág. $56-66$.

Avalos, K. R., \& S. S. (2003). Actividad antioxidante y contenido en fenoles totales en vinos de origin nacional. Facena.19:11-19. Disponible en: https://www.researchgate.net/publication/265159361 actividad antioxidante $y$ contenido en fenoles totales en vinos de origin nacional.

Brand-Williams, W. C. (1995). Use of free radical method to evaluate antioxidant activity. Lebensm. Qwiss.u - Technol, 28, 25 - 30.

Durán, D., \& Trujilllo, Y. (2008). Estudio comparativo del contenido fenólico de vinos tintos colombianos e importados. Vitae. Revista de la Facultad de Química y Farmacología, 15 (1):17-24.

Fernández-Pachón, M. T.-P. (2004). The antioxidant activity of wines determined by the DPPH method: influence of sample dilution and time. Talanta, 64: 501509.

Flanzy, C. (2003). Enología: Fundamentos científicos y tecnológicos. España: Mundi-Prensa. Madrid, $783 \mathrm{p}$.

Frankel, E. W. (1995). Principal Phenolic Phytochemicals in Selected California Wines and Their Antioxidant Activity in Inhibiting Oxidation of Human LowDensity Lipoproteins. J. Agric. Food Chem.

Hamasaka, T. K. (2004). Antioxidant activity and constituents of propolis collected in various areas of Japan. Japan: Food Sc. Techno Res, pag. 86 - 92.

Jáuregui, M. A. (2007). Evaluación de la actividad antioxidante y contenido de compuestos fenólicos en vinos producidos en Perú. Soc Quím Perú, 73, № 1 $(30-40)$.

Ketter, R. (2008). Caracterización de la composición fenólica de vinos comerciales del cv. Cabernet Sauvignon provenientes de tres valles de Chile, de las vendimias 2002 y 2003. Memoria Ingeniero Agrónomo. Universidad de Chile, Facultad de Ciencias Agronómicas, Santiago, Chile. 51 p. 
Landrault, N. P. (2001). Antioxidant capacities and phenolics levels of French wines from different varieties and vintages. Journal of Agricultural and Food Chemistry, 49: 3341- 3348.

lanzy, C. (2003). Enología: Fundamentos científicos y tecnológicos. España: Ediciones Mundi-Prensa. Madrid. 783p.

Leyva, D. E. (2009). Determinación de antocianinas, fenoles totales y actividad antioxidante en licores y fruto de mora. Universidad tecnológica de la mixteca Huajuapan de león, Oaxaca,México.

Llobera, A. (2004). "Obtención de antioxidants naturals a partir de subproductes del procés de vinificació del raïm autòcton Manto negro". Conselleria d'Agricultura i Pesca, Quaderns d'Investigació, Vol. 1.

Muñoz. (2006). reporta concentraciones entre 3,2 a 3,2 g equivalente de procianidina/L, en el cv. Carménère utilizando la metodología de Bate-Smith.

Muñoz A, F. A. (2007). Evaluacion de la actividad antioxidante y contenido en fenoles totales en vinos producidos en perú. Sociedad quimica del perú, 73 (1): $30-40$.

Narváez, J. (2010). Comparación de tres métodos de medición de taninos totales y su relación con la astringencia y el amargor percibido por un panel de degustación especializado. Universidad de Chile, Facultad de Ciencias Agronómicas.Santiago, Chile. 97 p.

Aguilar, T. (2015). Comparación de la capacidad antioxidante de mostos y vinos tintos del valle del Itata, Chile. Departamento de Agroindustrias, facultada de ingeniería Agricula, universidadde concepción, chillan, chile.

Alén-Ruiz, F. G.-F.-L.-C.-G. (2009). "influence of major polyphenols on antioxidant activity in mencía and brancellao red wines". food chemistry, vol. 113, pgs. 53 -60 .

Arteaga S., C. J. (2016). Análisis comparativo del contenido de taninos en vinos comerciales de Tarija, Bolivia. Ciencia Sur Vol. 2. № 2. ISSN 2518 - 4792, Pág. $56-66$.

Avalos, K. R., \& S. S. (2003). Actividad antioxidante y contenido en fenoles totales en vinos de origin nacional . Facena.19:11-19. Disponible en: https://www.researchgate.net/publication/265159361 actividad antioxidante y contenido en fenoles totales en vinos de origin nacional.

Brand-Williams, W. C. (1995). Use of free radical method to evaluate antioxidant activity. Lebensm. Qwiss.u - Technol, 28, 25 - 30.

Durán, D., \& Trujilllo, Y. (2008). Estudio comparativo del contenido fenólico de vinos tintos colombianos e importados. Vitae. Revista de la Facultad de Química y Farmacología, 15 (1):17-24.

Fernández-Pachón, M. T.-P. (2004). The antioxidant activity of wines determined by the DPPH method: influence of sample dilution and time. Talanta, 64: 501509. 
Flanzy, C. (2003). Enología: Fundamentos científicos y tecnológicos. España: Mundi-Prensa. Madrid, $783 \mathrm{p}$.

Frankel, E. W. (1995). Principal Phenolic Phytochemicals in Selected California Wines and Their Antioxidant Activity in Inhibiting Oxidation of Human LowDensity Lipoproteins. J. Agric. Food Chem.

Hamasaka, T. K. ( 2004). Antioxidant activity and constituents of propolis collected in various areas of Japan. Japan: Food Sc. Techno Res, pag. 86 - 92.

Jáuregui, M. A. (2007). Evaluación de la actividad antioxidante y contenido de compuestos fenólicos en vinos producidos en Perú. Soc Quím Perú, 73, № 1 $(30-40)$.

Ketter, R. (2008). Caracterización de la composición fenólica de vinos comerciales del cv. Cabernet Sauvignon provenientes de tres valles de Chile, de las vendimias 2002 y 2003. Memoria Ingeniero Agrónomo. Universidad de Chile, Facultad de Ciencias Agronómicas, Santiago, Chile. $51 \mathrm{p}$.

Landrault, N. P. (2001). Antioxidant capacities and phenolics levels of French wines from different varieties and vintages. Journal of Agricultural and Food Chemistry, 49: 3341- 3348.

lanzy, C. (2003). Enología: Fundamentos científicos y tecnológicos. España: Ediciones Mundi-Prensa. Madrid. 783p.

Leyva, D. E. (2009). Determinación de antocianinas, fenoles totales y actividad antioxidante en licores y fruto de mora. Universidad tecnológica de la mixteca Huajuapan de león, Oaxaca,México.

Llobera, A. (2004). "Obtención de antioxidants naturals a partir de subproductes del procés de vinificació del raïm autòcton Manto negro". Conselleria d'Agricultura i Pesca, Quaderns d'Investigació, Vol. 1.

Muñoz. (2006). reporta concentraciones entre 3,2 a 3,2 g equivalente de procianidina/L, en el cv. Carménère utilizando la metodología de Bate-Smith.

Muñoz A, F. A. (2007). Evaluacion de la actividad antioxidante y contenido en fenoles totales en vinos producidos en perú. Sociedad quimica del perú, 73 $(1): 30-40$.

Narváez, J. (2010). Comparación de tres métodos de medición de taninos totales y su relación con la astringencia y el amargor percibido por un panel de degustación especializado. Universidad de Chile, Facultad de Ciencias Agronómicas.Santiago, Chile. 97 p.

Radovanovie, A. (2010). Determinación de flavonoides totales en vinos cv. Carménère. Molecules, 15. 4213.

Ribéreau-Gayon, P. G. (1998). Dosage des tannins du vin rouge et. Chim. Anal, 179 $-260$.

Rodrigo, S., Giovana, E., Candy, R., María, F., \& Rosario, R. (2011). Compuestos fenólicos, actividad antioxidante, contenido de resveratrol y componentes del 
aroma de 8 vinos peruanos. Departamento de Ciencias Farmacéuticas: Facultad de Ciencias y Filoso.

Rodríguez, R. G. (2005). Actividad antioxidante y composición fenólica en vinos de Castilla- La Mancha. Revista de Tecnología Higiene Alimentaria, 362 (5):128:132.

Salazar R, E. G. (2011). Compuestos fenólicos, Actividad antioxidante y contenido de resveratrol y componentes del aroma de 8 vinos peruanos. La sociedad quimica del perú. Disponible en: https://www.scielo.org.pe/scielo.php?s script=scri_arttex y pid=s1810-634x2011000200006\$script=scri_isot, 77 (2): $135-143$.

Simonetti, P. P. (1997). Polyphenol content and total antioxidante potential of selected Italian wines. Journal of Agricultural and Food Chemistry, 45: 11521155.

Singleton, V., Orthofer, R., \& Lamuela-Raventos, R. (1999). Analysis of total phenols and other oxidation substrates and antioxidants by means of Folin - Ciocalteu reagent. Methods Enzymol, 299, 152 - 178.

Soto, H. (enero - marzo 2007 vol 30 no. 1 Chapingo). "Actividad antioxidante de flavonoides del tallo de orégano mexicano (lippia graveolens hbk var. Berlandieri Schafer)". Fitotecnia Mexicana, 43 - 49.

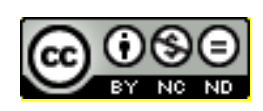

Determinación de la Actividad Antioxidante, contenido de Fenoles Totales, Taninos Totales y Flavonoides Totales del Hidromiel de Sauco (sambucus peruviana) de Cuatro Empresas del Distrito de Talavera en el año 2019 (Jorge Wilmer Elías - Silupu) Por Revista Sendas se encuentra bajo una Licencia Creative Commons-No Comercial-Sin Derivadas 3.0 Uported. 